\title{
Contribution à l'étude faunistique et écologique des simulies (Diptera, Simuliidae) du Maroc I. Une nouvelle espèce du Haut-Atlas : Simulium (Nevermannia) toubkal n. sp.1
}

\author{
A. Bouzidi2 \\ J. Giudicelli3
}

Mots clés : Diptera, Simuliidae, nouvelle espèce, Haut-Atlas, Maroc.

Une espèce nouvelle de Simulium Latreille s.l. appartenant au sous-genre Nevermannia Enderlein est décrite du HautAtlas (Maroc). L'espèce nouvelle, S. (N.) toubkal, est étroitement apparentée à $S$. (N.) rivosecchii (Contini, 1965), espèce connue seulement de Sardaigne (Italie). Les deux espèces sont bien distinctes des autres espèces connues de Nevermannia par la forme, la structure superficielle et l'insertion basale des filaments respiratoires nymphaux. Toutes les deux sont des especes de haute altitude et d'eau froide. S. (N.) toubkal a été récoltée dans le plus haut massif du Maroc (jbel Toubkal) entre 2300 et 3000 mètres d'altitude dans des torrents froids (température de l'eau 5 a $10^{\circ} \mathrm{C}$ en juillet).

\footnotetext{
Contribution to the faunistic and ecological study of the blackfles (Diptera, Simulidae) of Morocco. I. A new species from High Atlas: Simulitum (Nevermannia) totobkal $\mathrm{n} . \mathrm{sp}$.
}

Keywords : Diptera, Simuliidae, new species, High Atlas, Morocco.

A new species of Simulium Latreille s.l. belonging to the subgenus Nevermannia Enderlein is described from the High At las (Morocco). The new species $S$. (N.) toubkal is phylogenetically close to $S$. (N.) rivosecchit (Contini 1965 ) a species known only from Sardinia (Italy). Both are well differentiated from all other known species of the subgenus by the shape, the surface structure and the basal branching of gill filaments. Both are high altitude and cold water species. $S$. (N.) toubkal has been collected in the highest massif of Morocco (jebel Toubkal) between 2300 and 3000 metres a.s.l. in cold torrential streams (water temperature : $5-10^{\circ} \mathrm{C}$ in July).

\section{1. - Introduction}

Dans te cadre de l'action de recherche intégrée franco-marocaine AI 24-82 (" Ecologie des milieux aquatiques continentaux des pays méditerranéens $n)$ des récoltes d'invertébrés benthiques ont été systématiquement entreprises depuis 1982 sur les

1. Action de Recherche Integrée Franco-Marocaine AI 82-24.

2. Laboratoire d'Hydrobiologie, Faculté des Sciences, BP S 15 Marrakech, Maroc.

3. Laboratoire de Biologie Animale et Ecologie, Faculté des Sciences de Saint-Jéröme, Rue Henri Poincaré, 13397 Marseille Cédex 13, France. principaux réseaux hydrographiques du Haut-Atlas marocain, tant sur ceux du versant atlantique (oued Ourika, oued Tensift, oued Reghaya, oued Nfiss) que sur ceux du versant saharien (oued $Z \mathrm{i} z$, oued Dades, oued Todra, oued Mellah).

L'étude des nombreux prélèvements réalisés révèle un abondant matériel de Simuliidés où figurent plusieurs nouveautés faunistiques. La description d'une espèce crénobionte très caractéristique par son appareil respiratoire nymphal, Simulitum knidirii Giudicelli \& Thiery, a fait l'objet d'une récente publication (Giudicelli \& Thiery 1984). D'autres espèces, que nous croyons nouvelles pour la 
science, sont en cours d'étude. Dans le présent travail nous donnons la description complète et l'écologie d'une espèce nouvelle appartenant au sousgenre Nevermannia, trouvée aux altitudes supérieures dans des torrents du Haut-Atlas de Marrakech et pour laquelle nous proposons le nom de Simulium (Nevermannia) toubkal.

\section{2. - Description de Simulium (Never- mannia) toubkal n. sp.}

\section{1. - Matériel examiné}

L'holotype et les paratypes $\approx$, l'allotype et les paratypes $q$ sont extraits de nymphes mûres.

Holotype $\sigma$, allotype $Q, 11$ paratypes $\odot, 28$ nymphes, 9 larves, Maroc : assif n'Ouarzane (réseau supérieur du l'oued Nfiss) alt. $3000 \mathrm{~m}$, au niveau du refuge de Lepiney (massif du Toubkal), $31^{\circ} 5^{\prime} \mathrm{N} /$ $7^{\circ} 58^{\prime} \mathrm{W}, 21 . \mathrm{VII} .1985$.

5 paratypes $\odot, 16$ nymphes, 3 larves, Maroc : assif n'Ouarzane, au pied des cascades d'Irhoulidene (alt. $2800 \mathrm{~m}), 22$. VII.1985.

Certains spécimens récoltés dans la station supérieure de l'assif $n$ 'Ouarzane sont montés sur lame dans le baume du Canada; les autres exemplaires mentionnés sont conservés en alcool.

L'holotype, l'allotype, plusieurs paratypes et paedotypes sont déposés dans la collection J. Giudicelli (Marseille) ; plusieurs paratypes et paedotypes sont déposés dans la collection du laboratoire d'Hydrobiologie de la Faculté des Sciences de Marrakech; 5 paratypes $\sigma, 6$ nymphes, 4 larves sont déposés dans la collection de Zoologie de l'Institut Scientifique de Rabat.

\section{2. - Mâle}

\section{- Tête}

Clypeus brun sombre, portant de chaque côté de longs poils dressés clairs. Antennes jaunes gris à 11 articles; article basal brun; troisième article presque deux fois plus long que l'article suivant (fig. 1).

\section{- Thorax}

Mesonotum brun sombre, sans tache argentée, couvert d'une pilosité dorée brillante. Pleurites bruns ; membrane pleurale et katepisternum nus.

\section{- Pattes (fig. 3, 4, 5)}

Coxae et trochanters bruns. Fémurs jaunes, bruns à l'extrémité distale. Tibias jaunes dans la partie médiane, bruns aux extrémités et sur le bord supérieur. Tarses brun clair; basitarse III brun gris, aplati et très large (rapport longueur/largeur $=4,7$ ) ; calcipala et pédisulcus nets. Griffes des tarses munies d'une grosse dent basale (fig, 6).

\section{- Abdomen}

Jaune avec une large bande dorsale brune et une bande ventrale étroite.

\section{- Genitalia}

Coxite 3,5 a 4 fois plus long que large en vue ventrale (fig. 7,8$)$. Style long comme la moitié de la longueur du coxite ; extrémité distale élargie en forme de processus triangulaire interne saillant et portant l'épine terminale (fig. 9, 10). Plaque ventrale (fig. 11) subrectangulaire ou trapézoïdale, deux fois et demi plus large que haute; bords latéraux verticaux ; bord postérieur légèrement déprimé et rectiligne en son milieu, arrondi au niveau des angles ventrolatéraux ; bras courts, pointus et faiblement courbés vers l'intérieur; la zone centrale couverte de poils est large et n'atteint pas le bord antérieur de la plaque, elle n'est pas saillante en forme de carène, les soies latérales situées sur le bord postérieur sont plus longues et implantées sur de petits tubercules.

Plaque dorsale sclérifiée, circulaire ou en forme d'ampoule et brune sombre concolore (fig. 13, 14). Processus médian en forme d'une bande étroite échancrée à partir de son tiers distal en deux pointes divergentes (fig. 15). Paramères rectangulaires, portant des épaississements sclérifiés et une forte épine noire (fig. 12).

\section{3. - Femelle}

\section{- Tête}

Antennes brun jaunâtre. Clypeus parsemé de poils clairs. Front jaunâtre avec une tâche médiane brune. Région occipitale brune couverte de longs poils jaunes, parsemée de quelques poils sombres dressés. Mandibule : 32 à 35 dents du côté interne, 12 dents du côté externe : galea maxillaire munie de grosses dents pointues: 12 externes, 15 internes; palpe labial : troisième article brunâtre, dilaté, contenant une grosse vésicule sensorielle, dernier article presque aussi long que les deux précédents réunis ( $f i g$. 16) ; cibarium non armé. 

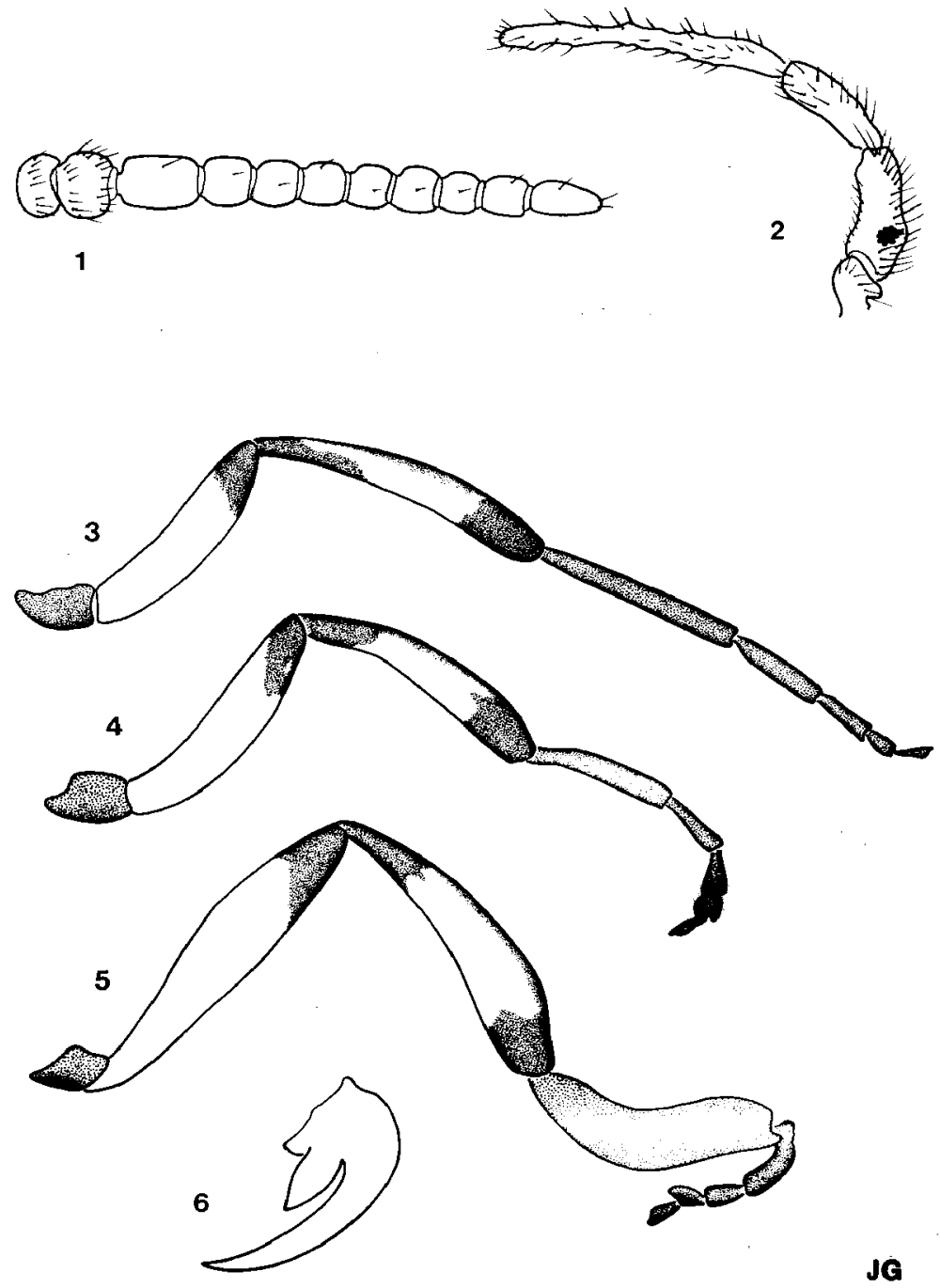

Fig. 1 à 6. - Simulium toubkal n. sp. Mâle.

1 : antenne. 2 : palpe maxillaire. 3 : patte 1.4 : patte II. 5 : patte III. 6 : griffe. 

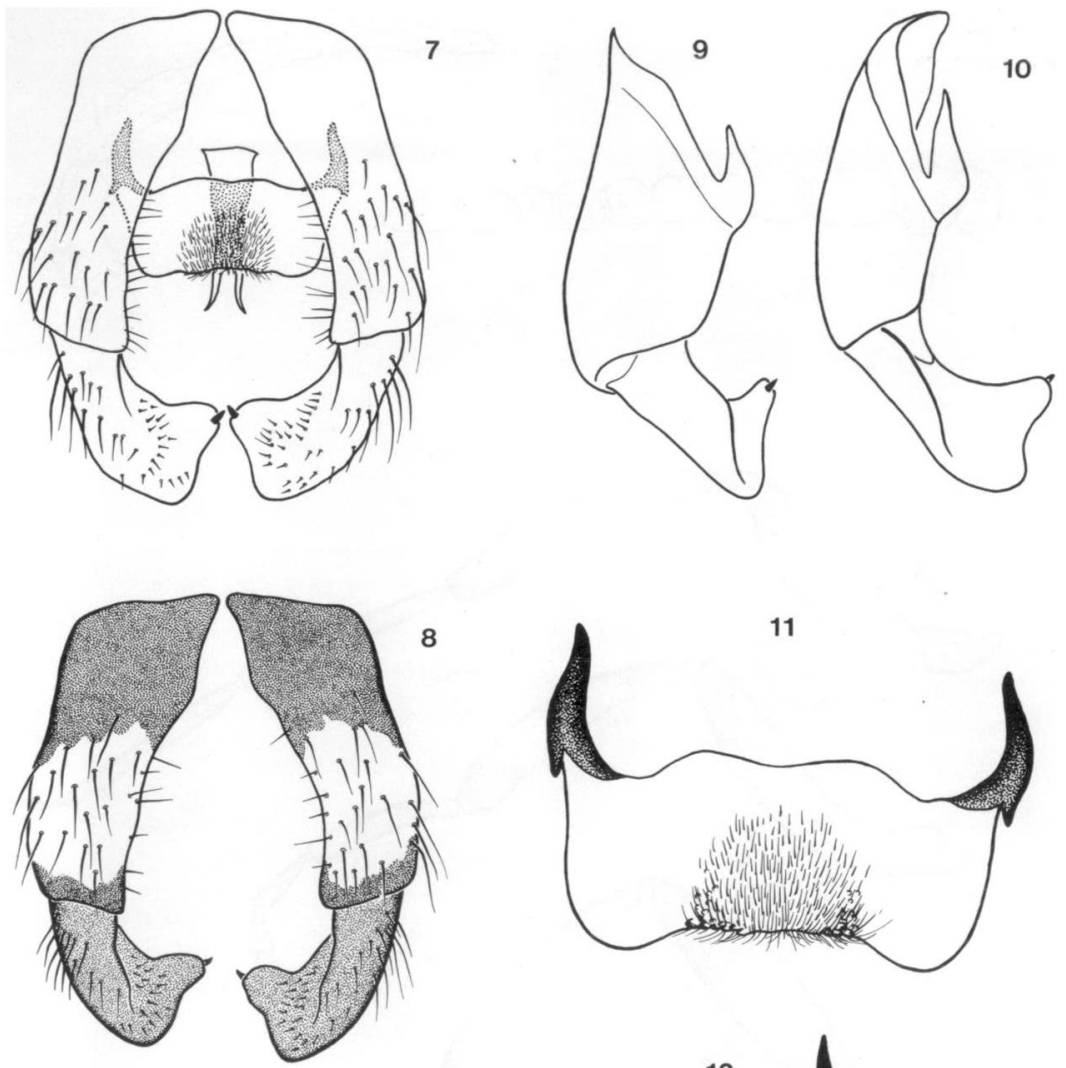

13

14
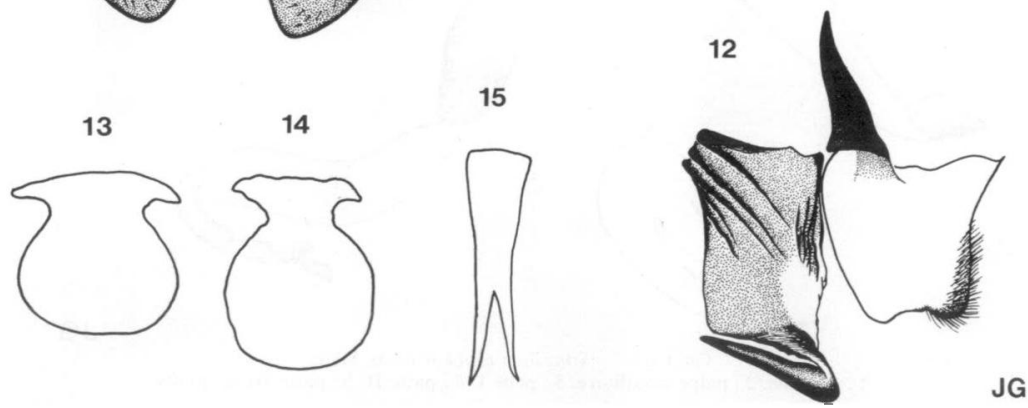

Fig. 7 à 15. - Simulium toubkal n. sp. Mâle.

7 : pièces génitales en vue ventrale. 8 : gonopodes en vue ventrale. 9 : style gauche de profil. 10 : style gauche en vue dorsolatérale. 11 : plaque ventrale. 12 : paramère et plaque ventrale de profil. 13,14 ; plaque dorsale. 15 : processus médian. 
- Thorax

Pronotum et pattes avec la même coloration que chez le mâle.

- Genitalia (fig. 17, 18, 19)

Aire médiane du sternite VIII bien sclérifiée et à contour net, constituée d'une grande plaque déprimée et presque ronde prolongée de 4 petites expansions latérales. Gonapophyses triangulaires, à extrémité arrondie ; bords internes sclérifiès, bien séparés l'un de l'autre; sur chaque gonapophyse 6 ou 7 soies. Furca pigmentée, branches latérales très écartées. Spermathèque brune, ovoïde, à large ouverture ; paroi pourvue d'une ornementation polygonale. Paraproctes courts et larges, présentant une petite saillie ventrale; cerques bruns, bord postéroventral arrondi, bord dorsal presque rectiligne.

\section{4. - Nymphe (fig. 20, 21)}

Longueur du corps (filaments respiratoires non compris) : 3,9-4,4 $\mathrm{mm}$. Tégument de la région dorsale du céphalothorax couvert de très nombreux petits tubercules arrondis et lisses (diamètre: 5-8 $\mu \mathrm{m})$.

- Trichomes simples atteignant une longueur de 150 à $260 \mu \mathrm{m}$, ceux du capuchon céphalique sont plus courts (95 à $110 \mu \mathrm{m}$ ) et au nombre de trois.

\section{- Appareil respiratoire (fig. 22, 23)}

Il comprend de chaque côté 4 filaments, presque aussi longs que le corps de la nymphe ( $\mathrm{L}$ : 3,6 - $4 \mathrm{~mm}$ ), groupés en un faisceau parallèle au plan du substrat sur presque toute sa longueur. Contrairement à la plupart des espèces du genre Nevermannia, les filaments ne sont pas disposés en 2 paires portées chacune par un pédoncule plus ou moins long mais tous les quatre sont insérés au même niveau sur un tronc épais porté par une saillie du thorax. On distingue deux filaments dorsaux contigus à la base, et deux filaments ventraux nettement séparés au niveau de leur insertion sur le tronc commun. Les filaments dorsaux sont un peu plus épais, dans leur partie proximale, que les filaments ventraux. Paroi du tronc basal craquelée, paroi des filaments annelée.

- Abdomen

De part et d'autre de la ligne médiane, on relève l'ornementation tégumentaire suivante (fig. 24).
Dorsalement : 2 soies fines sur le segment 1 ; $5-6$ soies robustes près du bord postérieur du segment II ; sur les segments III et IV, 4 gros crochets simples, une soie courte située en avant entre le $3^{e}$ et le $4^{e}$ crochet, 2 soies externes; sur les segments V à VIII, une bande antérieure d'épines plates passant latéralement à des écailles; sur $V, 3$ petites soies latérales ; sur IX, une épine terminale.

Ventralement : III et IV, 3 soies; V, 2 crochets bifides rapprochés et 2 soies; VI, 2 crochets bifides écartés; VII, un crochet simple et un crochet bifide à branches inégales.

- Cocon

Longueur : $6,6-7,3 \mathrm{~mm}$. Couleur jaune clair. Texture compacte. Forme aplatie ; bord antérieur renforcé et présentant un processus médian long et étroit ; la base du processus médian est soutenue par le renforcement du bord antérieur (fig. 21).

\section{5. - Larve}

Longueur: $7,5-8,4 \mathrm{~mm}$ (larve mûre). Coloration générale jaune clair, capsule céphalique jaunâtre. - Fronto-clypeus (fig. 25)

Jaune clair, rembruni le long du bord postérieur ; taches brun clair correspondant aux insertions musculaires, la tache médiane et les deux taches latérales postérieures étant plus foncées.

- Plaques épicraniales

Jaunâtres ; sourcil net ; une double tache sombre en arrière de l'ceil en contact avec le bord postérieur.

- Echancrure ventrale (fig. 26)

Forme subpentagonale ; sommet arrondi, dépassant légèrement la moitié de la distance comprise entre la base des apodèmes et le bord inférieur du submentum.

- Submentum (fig. 27)

Bord antérieur muni de 9 dents ; la dent médiane et les deux dents latérales sont les plus développées, la médiane étant la plus saillante ; bords latéraux avec 4 dents dont la première est la plus grande ; deux rangées latérales de 5-6 soies, les trois premières étant divisées à l'apex.

\section{- Antennes}

Coloration jaune très clair; longueur : $550 \mu \mathrm{m}$; l'extrémité du deuxième article at teint l'extrémité de la hampe des prémandibules. 

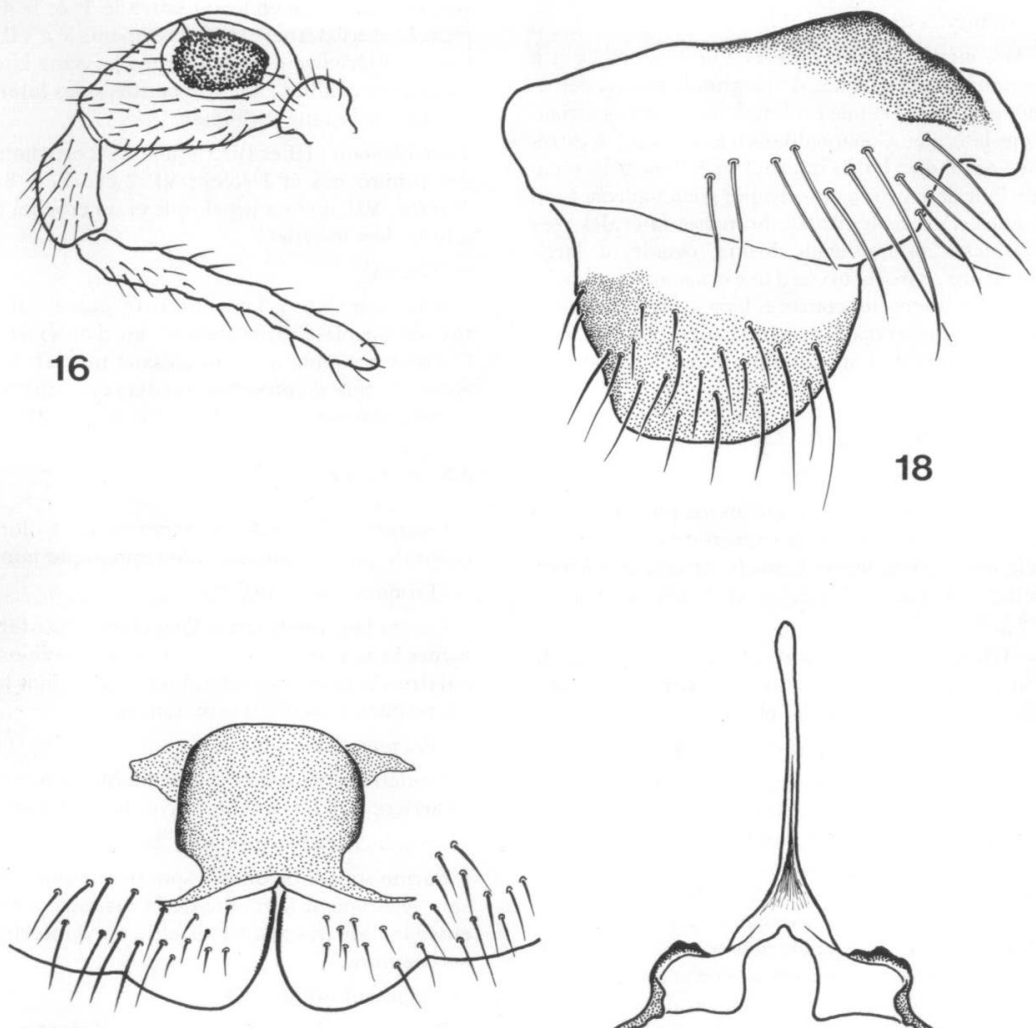

17

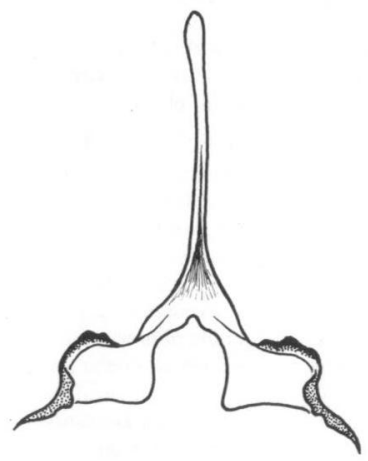

19

JG

FIG 16 à 19. - Simulium toubkal n. sp. Femelle.

16 : palpe maxillaire. 17 : genitalia. 18 : paraprocte et cerque. 19 ; furca. 

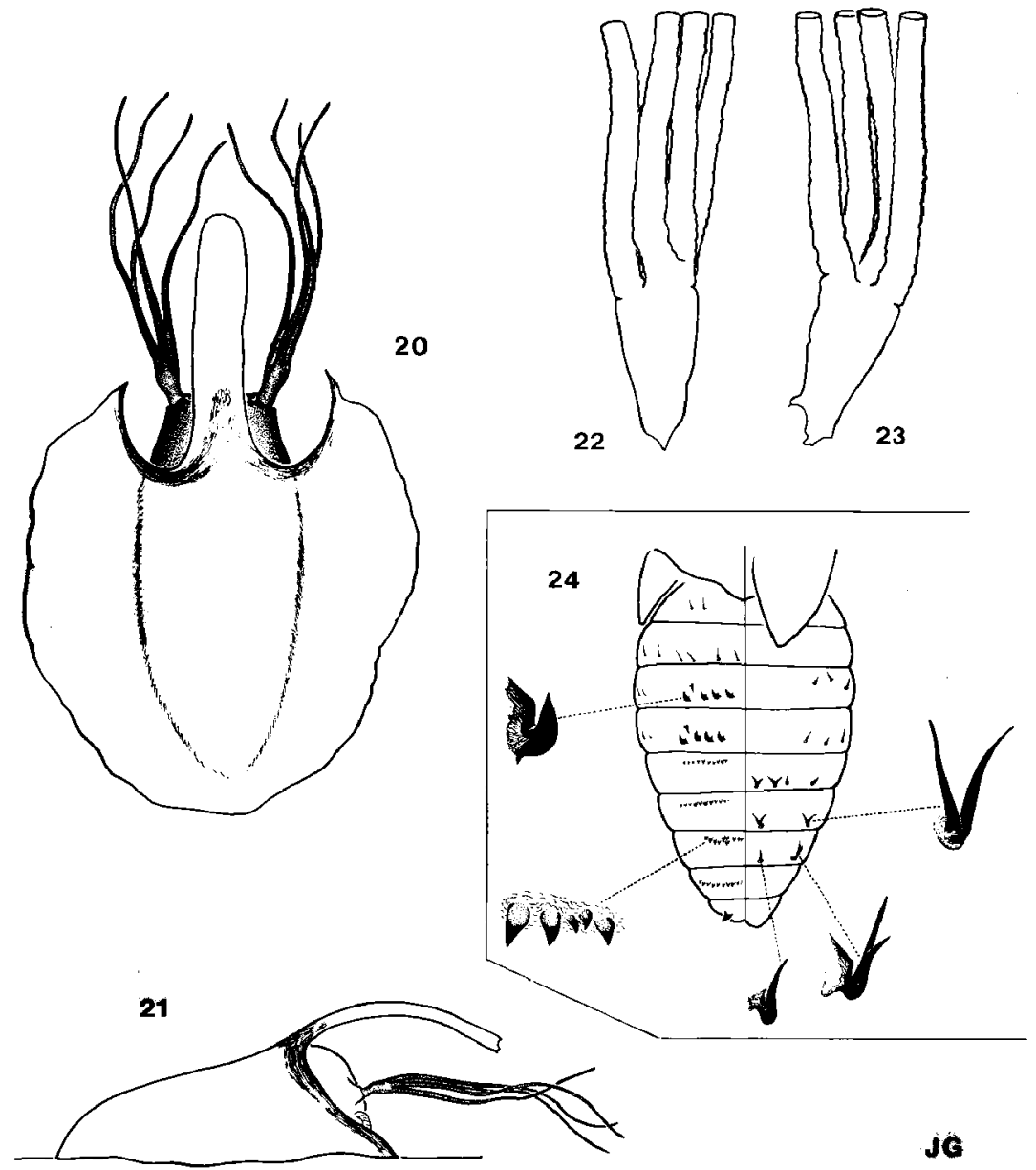

FIG. 20 à 24 . - Simulium toubkal n. sp. Nymphe.

20: vue dorsale. 21 : vue de profil. $22:$ filaments respiratoires en vue dorsale

23 : filaments respiratoires en vue ventrale. 24 : ornementation tégumentaire de l'abdomen. 
- Prémandibules

29 à 31 baguettes falciformes.

- Mandibules (fig. 28)

Rangée $b t$ composée de 7 dents ; processus $t p$ comprenant une grosse dent suivie d'un groupe de 5-6 petites dents égales.

- Abdomen

Une paire de grosses papilles ventrales coniques. Branchies rectales ramifiées avec 9 à 14 branches secondaires de même taille que la branche terminale. Sclérite anal ( $f$ ig. 29 ) formé de 4 bras subégaux : la cuticule abdominale située de chaque côté du sclérite porte de nombreuses soies courtes transparentes. Disque postérieur formé de 75 à 82 rangées de 12 à 15 crochets.

\section{3. - Discussion sur la position taxo- nomique de Simulium toubkal}

A tous les stades de son développement, et en particulier au niveau des genitalia mâles, $S$. toubkal présente les caractères morphologiques qui sont conformes à ceux de Simulium (Nevermannia) groupe vernum.

Parmi les espèces de Nevermannia, celle dont l'espèce marocaine se rapproche le plus est $S$. $(N)$ rivosecchii (Contini), en démique de Sardaigne, décrite en 1965 sous le nom générique de Eusimulium; dans sa faune des Simuliidae d'Italie, Rivosecchi (1978) la décrit sous le nom de Cnetha rivosecchii.

Les affinités morphologiques et phylogénétiques les plus nettes qui existent entre les deux espèces apparaissent dans les caractères suivants :

- basitarse III du mâle très élargi,

- gonocoxite des mâles long en vue ventrale ; gonostyle se terminant par un processus interne très développé,

- plaque ventrale de l'édéage très large et munie de bras très courts,

- forme de cerques, des paraproctes, des gonapophyses, de la furca chez les femelles,

- forme du submentum, de l'échancrure ventrale de la capsule céphalique et du processus $t p$ chez les larves,

- forme, structure superficielle et disposition des filaments respiratoires chez les nymphes.

Le dernier caractère mentionné traduit le mieux l'originalité des deux espèces et permet de les différencier immédiatement des autres espèces connues de Nevermannia.

L'espèce sarde et l'espèce marocaine, bien qu'apparaissant taxonomiquement isolées au sein de ce sous-genre, sont cependant bien distinctes l'une de l'autre par les points suivants :

\section{CHEZ LE MÁLE}

- bord postérieur de la plaque ventrale de l'édéage profondément déprimé chez $S$. rivosecchii, à peu près rectiligne chez $S$. toubkal; bras de la plaque vent rale plus courts chez $S$. rivosecchii; aire piligère de la plaque ventrale plus étroite, plus haute et formant une carène chez $S$. rivosecchii,

- plaque dorsale de l'édéage arrondie et bien pigmentée chez $S$. toubkal, plutôt rectangulaire et dépigmentée chez l'autre espèce.

\section{Chez LA FEMELLe}

- aire centrale du $8^{e}$ sternite abdominal plus nette et de forme bien caractéristique chez $S$. toubkal.

\section{CHEZ LA NYMPHE}

- faisceau des filaments respiratoires incliné vers le bas en arc de cercle et venant au contact du substrat chez $S$. rivosecchii; faisceau parallèle au substrat chez $S$. toubkal,

$-4^{e}$ filament (le plus ventral) plus grèle et inséré sur le tronc basal plus en arrière que les trois autres chez $S$. rivosecchii; les 4 filaments sont insérés au même niveau chez $S$. toubkal,

- trichomes thoraciques et céphaliques ramifiés chez $S$. rivosecchii, simples chez l'aut re espece,

- cocon dépourvu de processus médio-dorsal chez $S$. rivosecchii.

\section{Chez La LaRve}

- fronto-clypeus brunâtre chez $S$. rivosecchii, jaune chez $S$. toubkal,

- branchies anales portant 3 filaments secondaires chez $S$. rivosecchii, 9 à 14 filaments secondaires chez S. toubkal.

\section{4. - Données écologiques}

Simulium toubkal est une espèce sténotherme froide de haute altitude. Elle vit dans les torrents constituant le secteur initial des réseaux hydrographiques issus du flanc nord du plus haut massif marocain, le jebel Toubkal (alt. $4167 \mathrm{~m}$ ). Presque 


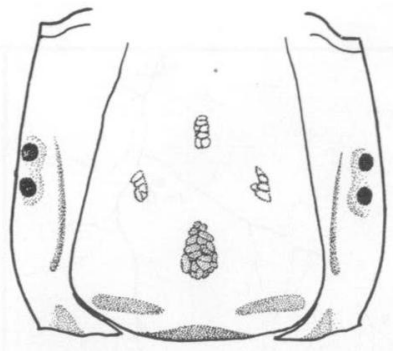

25
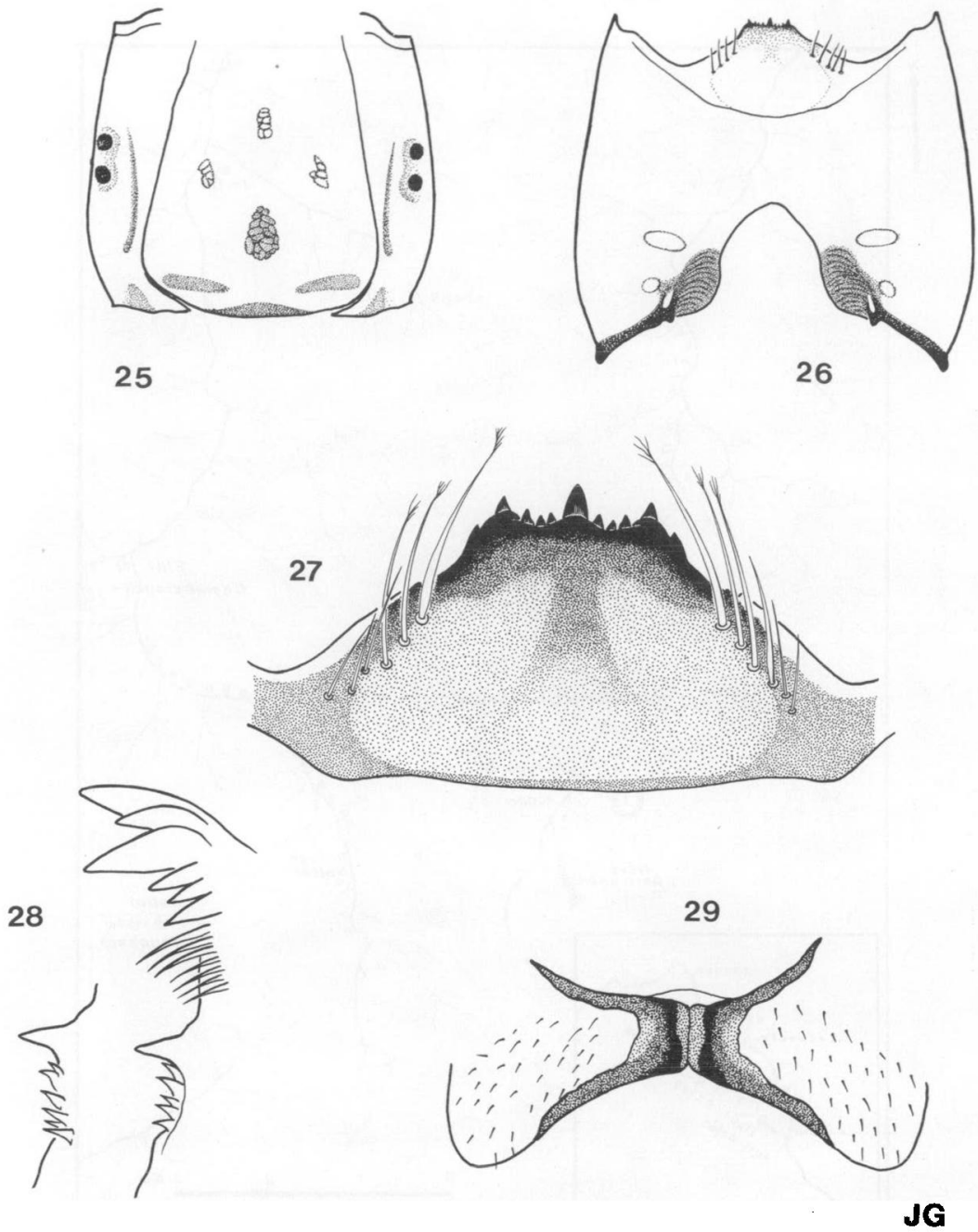

Fig. 25 à 29 . - Simulium toubkal n. sp. Larve.

25 : capsule céphalique en vue dorsale. 26 : capsule céphalique en vue ventrale. 27 : submentum. 28: extrémité de la mandibule. 29 : sclérite anal. 


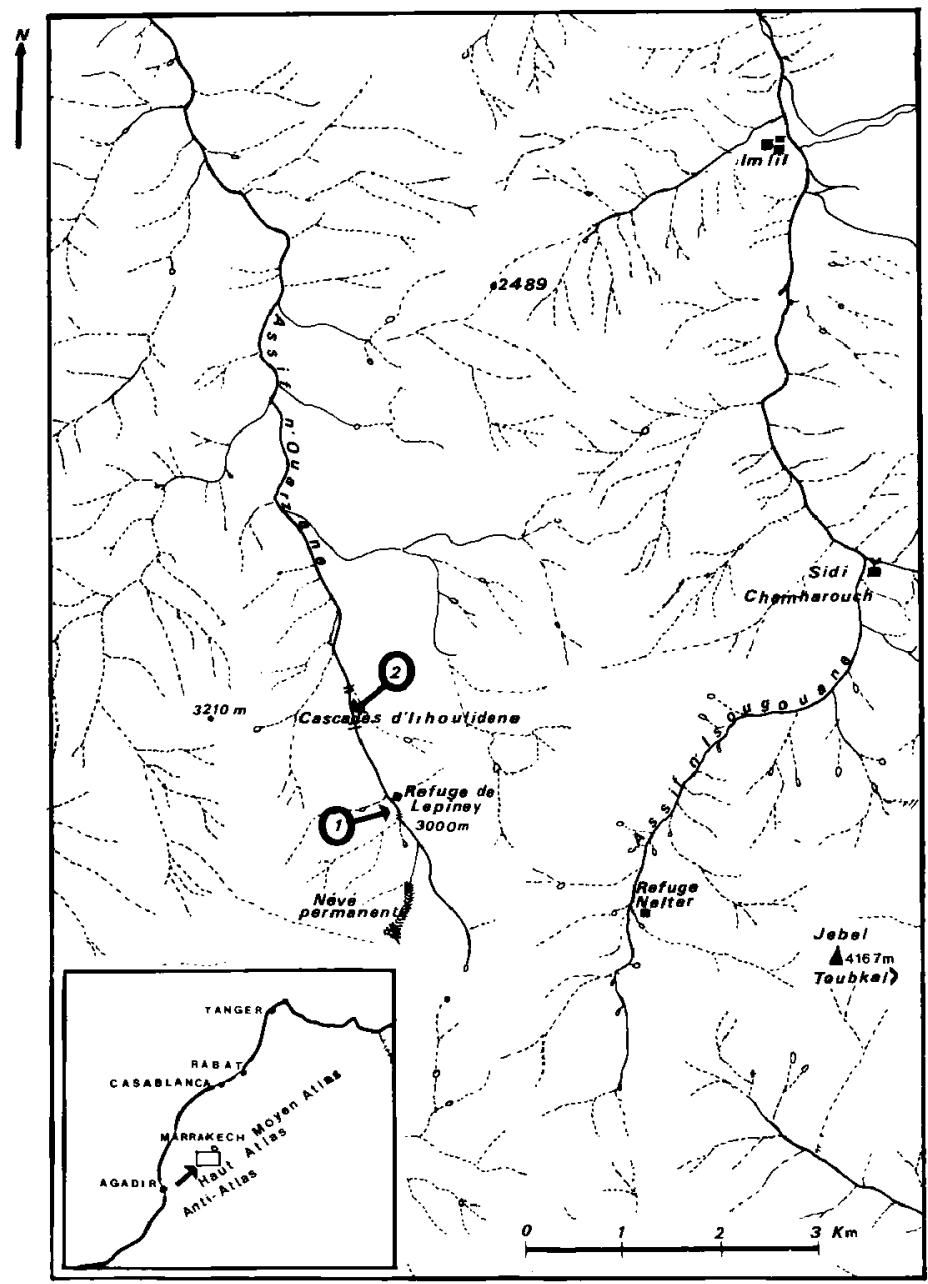

FiG. 30. - Simulium toubkal n. sp., situation géographique des stations des types (1 et 2). 

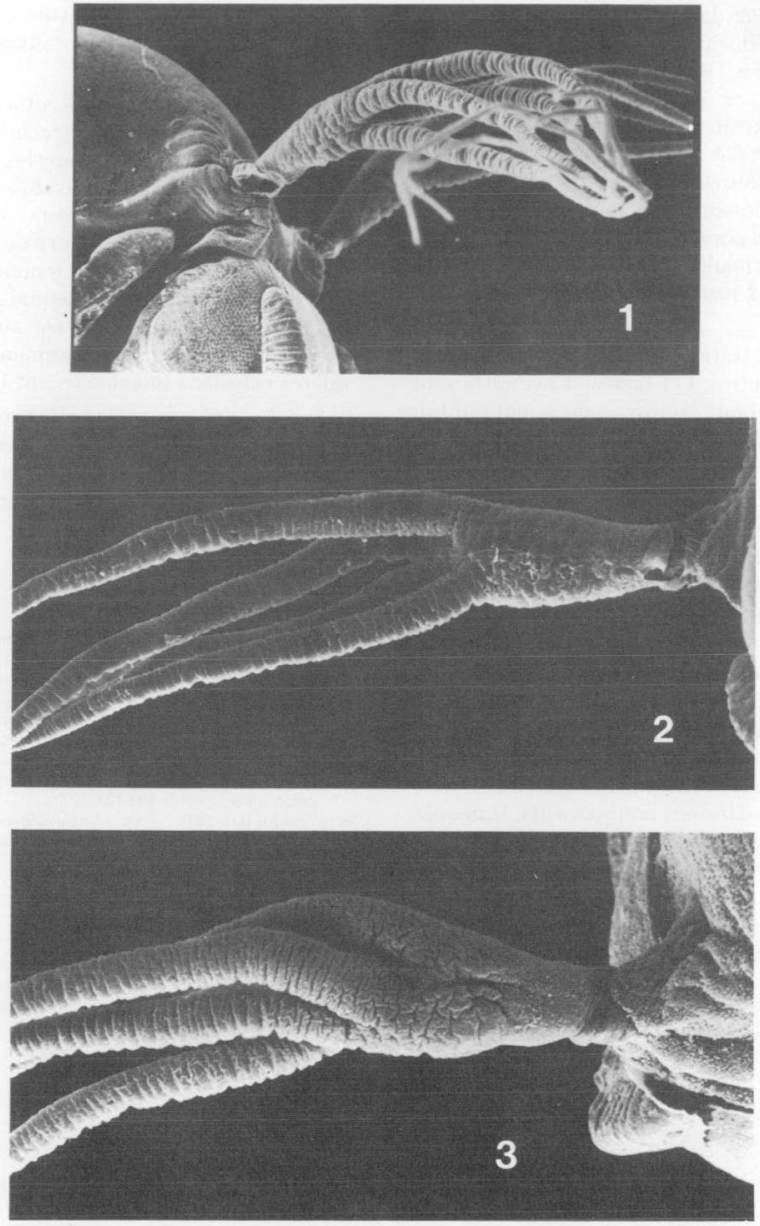

Planche I. - (microscopie à balayage) - Simulium toubkal n. sp. Nymphe. 1 : filaments respiratoires. 2 : filaments respiratoires en vue ventrale, 3 : filaments respiratoires en vue dorsale. 
tout le matériel larvaire et nymphal a été récolté à la fin de Juillet 1985 dans le cours supérieur de l'assif n'Ouarzane, affluent de l'oued Nfiss. La première station est située à 3000 mètres d'altitude, au bas de la cascade issue du névé permanent du jebel Adrar n'Ouanoukrim, dans des eaux dont la température était de $5^{\circ} \mathrm{C}$ à 9 heures et de $7^{\circ} \mathrm{C}$ à 15 heures le 21.7.1985. Sur le même cours d'eau, à $1 \mathrm{~km}$ en aval de la station précédente, nous avons trouvé le même jour une population de $S$, toubkal au pied des cascades d'I rhoulidene (alt. $2800 \mathrm{~m}$ ) dans une eau à $9,6^{\circ} \mathrm{C}$ (à 12 heures), la température de l'air étant de $18^{\circ} \mathrm{C}$.

Dans ces deux stations le torrent a une largeur moyenne de 4 mètres. Les larves et nymphes sont fixées sur les cailloux au niveau du replat qui fait suite à la cascade ; dans cet habi tat Simulium toub$k a l$ vit en compagnie des formes aquatiques d'une espèce inédite de Simulium Latreille s.s. du groupe monticola dont la description est en cours.

Quatre dépouilles nymphales ont été récoltées en Août 1985 dans l'assif n'Isougouane (cours supérieur de l'oued Reghaya) en armont du marabout de Sidi Chamharouch, à $2300 \mathrm{~m}$ d'altitude.

A la même époque nous avons réalisé d'autres prospections sur l'assif n'Ouarzane et sur d'autres torrents de haute altitude sur le flanc nord du massif du Toubkal et en particulier sur le réseau supérieur de l'oued Reghaya en amont l'Imlil. S. toub$k a l$ ne figure pas dans nos prélèvements, le peuplement simulidien de ces stations étant représenté par Simulium (Nevermannia) cryophilum et par S. (Tetisimulium) bezzit.

La quatrième station connue de $S$. toubkal (récolte de M. Mohati, le 7.VII.1984 : 8 nymphes, 3 larves) est un ruisseau du réseau supérieur de l'oued Ourika situé à 2800 mètres d'altitude près de l'azibl Likemt ( $\left.31^{\circ} 7 \mathrm{~N} / 7^{\circ} 49^{\prime} \mathrm{W}\right)$. Le ruisseau, long d'un kilor mètre environ, large de 1 à 2 mètres est alimenté par une source rhéocrène.

Le caractère alticole et microtherme définit aussi l'écologie de Simulium rivosecchii qui vit entre 800 et 1500 mètres d'altitude dans les secteurs les plus élevés des massifs cristallins du nord de la Sardaigne, avec pour habitat les petits cours d'eau permanents froids où la température de l'eau ne dépasse pas $10^{\circ} \mathrm{C}$. L'espèce sarde est monovoltine et termine son développement préimaginal en mai (Contini 1965); l'espèce marocaine est aussi monovoltine mais se développe plus tardivement puisque les premières éclosions imaginales ont lieu en Juillet.

Ainsi ces deux espèces constituent au sein du sousgenre Nevermannia et du groupe vermum une lignée méridionale ouest-méditerranéenne, orobionte et sténotherme, à distribution géographique limitée.

Travaux cités

Contini (C.). 1965, -- Descrizione e cenni biologici su una nuova specie di simulide del gruppo \& latipes * della Sardegna : Eusimulium rivosecchii n. sp. (Diptera, Nematocera). Riv. Parass, 26. 277.289 .

Giudicelli (J.) \& Thiery (A.). 1985. - About a peculiar type of rheocrene spring in the High Atlas (Morocco). Description of a simuliid characteristic of this habitat, Simulium (Crenosimulium n. sp.) knidinii n. sp. (Diptera, Simulitdae). Bull. zool. Mus. Univ. Amsterdam, 10 (15) 109-123.

Rivosecchij (L.). 1978. - Simulitdae Diptera Nematocera. Fauna d'Italia, vol. XII. Edizioni Calderini Bologna, 533 p.

1. Terme berbère désignant un groupe de bergeries en montagne. 